\title{
Current trends of laser technology in dermatology - A foreword from the JSD Editorial Office
}

I

$\mathrm{t}$ is a great pleasure to present Journal of Surgical Dermatology's first ever themed issue, "Lasers in dermatology: Current status". The themed issue contains scientific content that focus on highly relevant and important topics in the area of laser and light-based treatment in dermatology. Our Guest Editor, Dr. Nasrin Saki from the Department of Dermatology, Shiraz University of Medical Sciences, Iran, has been a pillar of support and it has been an honor to be able to work with such a talented laser expert.

The first lasers used to treat skin problems were introduced over 40 years ago. Recently, major advances in laser technology have revolutionized its use for dermatological conditions, hence being well-versed with this field is essential. For a dermatologist, lasers have become indispensable devices to modulate cutaneous biology, both in treating skin disorders and providing tangible cosmetic alterations to the skin.

The word "laser" originated as an acronym for "light amplification by the stimulated emission of radiation". Since the development of first laser by physicist Theodore H Maiman in 1960, it gained widespread acceptance in dermatology. Now, it is used for the treatment of numerous cutaneous conditions including pigmented and vascular lesions, tattoos, scars, unwanted hair and aging skin.

The principles of laser energy is based on the unique properties of laser light itself and the complex laser-tissue interactions. By allowing precise tissue destruction, laser light can be focused onto small spot sizes. Some light can be absorbed, reflected, transmitted or scattered when a laser encounters skin surface at each layers. With fewer session and minimum therapy time, various laser treatment options have proven effective to permanently remove unwanted pigmented lesions.

There are several types of lasers used in skin laser surgery. Older laser technologies, such as the first $\mathrm{CO}_{2}$ lasers which operated using a continuous wave $(\mathrm{CW})$ and argon, have been replaced by yellow-light quasi-CW and pulsed laser therapies. Major advances in laser technology have changed its use in the treatment of many skin conditions and congenital defects in the last 20 years. These include vascular and pigmented lesions, tattoo removal, reducing scars and wrinkles. There is an extensive range of laser and light technologies available for skin resurfacing and rejuvenation nowadays. The popular procedure is called lasabrasion, which is also known as laser peel, or laser vaporization. The technique directs short, concentrated pulsating beams of light at irregular skin, precisely removing skin layer by layer.

The number of devices approved to treat the appearance of cellulite has also grown since last year. Today, different kinds of non-surgical body-contouring modalities, including cryolipolysis, radiofrequency (RF), low-level laser therapy (LLLT), and high-intensity focused ultrasound (HIFU) are available for reducing the volume of subcutaneous adipose tissue or cellulite. Each and every procedure has particular mechanisms for stimulating apoptosis or necrosis adipose tissue.

According to PubMed and Google Scholar, the number of laser-based articles published since 2015 are quite high, close to 134,104 articles. These include publications on dermatologic laser surgery, laser treatment for pigmentation and vascular lesions. From JSD's article statistics, we consistently received a number of laser-based article submissions, mainly on laser treatment and laser therapy. On an average, we have found that at least two articles submitted for an issue were laser-based research. Topics on laser treatment studies have also garnered interest from the JSD readership: almost $20 \%$ of the total views for JSD's articles published online are for laser-based articles.

Authors who have contributed to this themed issue discussed the various types of lasers, the application of lasers in dermatology and also the alternative uses of lasers in medicine. In this themed issue, Dr. Saki has written an interesting editorial about picosecond laser applications in aesthetic dermatology. An original research article by Dr. Ghazala Butt and her co-workers from Pakistan's King Edward Medical University, which discusses the efficacy and safety of the intense pulse light (IPL) therapy's modes of action in the treatment of acne vulgaris, is also featured in this themed issue.

Besides those, two other articles on laser surgery are also published, and both of these articles focus on facial laser surgery. One is an author's perspective on the merits and demerits of lasers for specific facial conditions, while the other is a case report which explains how periocular syringomas were successfully treated with fractional $\mathrm{CO}_{2}$ 


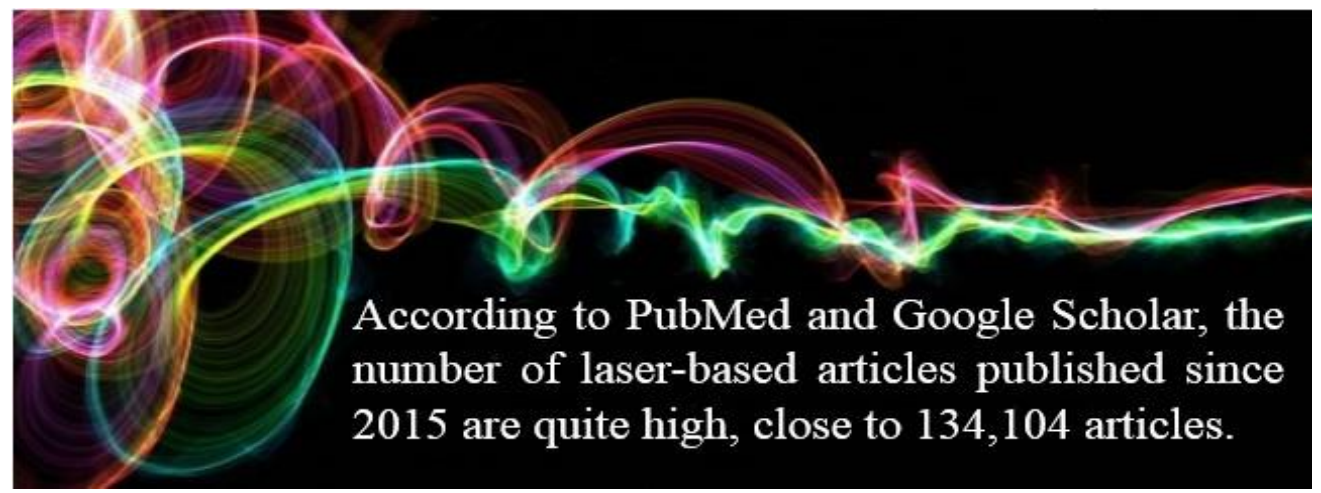

laser. On another noteworthy subject, treating hyperpigmentation disorders, especially melasma, with laser is a popular procedure by dermatologists, and Brazilian dermatologist Dr. Juliana Merheb Jordão and her co-author describe the treatment of melasma using energy-based devices. They elaborate on how cases unresponsive to alternative treatment modalities such as topical bleaches or chemical peels should consider laser therapy.
To sum up, laser treatment modalities are very important in medicine, and their uses in dermatology are becoming widespread. Dermatologists can better understand laser devices and explore their use confidently by gaining a thorough knowledge on the technology. We hope that this month's issue keeps you informed on the latest techniques, trends and technologies in the growing field of light and laser therapy. 